\title{
STRIPE-LIKE INHOMOGENEITIES, CARRIERS, AND BCS-BEC CROSSOVER IN THE HIGH-T $C$ CUPRATES
}

\author{
J. ASHKENAZI \\ Physics Department, University of Miami \\ P.O. Box 248046, Coral Gables, FL 33124, U.S.A.
}

\begin{abstract}
Considering both "large- $U$ " and "small- $U$ " orbitals, it is found that the carriers of the high- $T_{c}$ cuprates are polaron-like "stripons" carrying charge and located on stripe-like inhomogeneities, "quasi-electrons" carrying charge and spin, and "svivons" carrying spin and lattice distortion. This is shown to result in the observed anomalous spectroscopic and transport properties of the cuprates. Pairing results from transitions between pair states of stripons and quasi-electrons through the exchange of svivons, and a crossover occurs between BCS and Bose-Einstein condensation behaviors.
\end{abstract}

\section{Introduction}

Theoretical calculations $[1,2]$, and a variety of experimental data [3] support the assumption that the high- $T_{c}$ cuprates are characterized by dynamical stripe-like inhomogeneities, where narrow charged stripes form antiphase domain walls separating wider antiferromagnetic (AF) stripes. Experimental observations have been pointing to the presence of both itinerant and almost localized (or polaron-like) carriers in the cuprates. Firstprinciples calculations [4] support an approach based on the existence of both large- $U$ and small- $U$ orbitals in the vicinity of the Fermi level $\left(E_{\mathrm{F}}\right)$.

The fermion creation operator of a small- $U$ electron in band $\nu$, spin $\sigma$ (which can be assigned a number \pm 1 ), and wave vector $\mathbf{k}$ is denoted here by $c_{\nu \sigma}^{\dagger}(\mathbf{k})$. The creation operators of the large- $U$ electrons in the $\mathrm{CuO}_{2}$ planes are expressed using the "slave-fermion" method [5]. Such an electron in site $i$ and spin $\sigma$ is created by $d_{i \sigma}^{\dagger}=e_{i}^{\dagger} s_{i,-\sigma}$, if it is in the "upper-Hubbard-band", and by $d_{i \sigma}^{\prime \dagger}=\sigma s_{i \sigma}^{\dagger} h_{i}$, if it is in a Zhang-Rice-type "lower-Hubbard-band". 
Here $e_{i}$ and $h_{i}$ are ("excession" and "holon") fermion operators, and $s_{i \sigma}$ are ("spinon") boson operators. These auxiliary operators have to satisfy the constraint: $e_{i}^{\dagger} e_{i}+h_{i}^{\dagger} h_{i}+\sum_{\sigma} s_{i \sigma}^{\dagger} s_{i \sigma}=1$.

Further details on results presented here are given elsewhere [6].

\section{Auxiliary Space and Fields}

An auxiliary space is introduced within which a chemical-potential-like Lagrange multiplier imposes the constraint on the average. The projection of observable quantities to the physical space is achieved by expressing them as combinations of Green's functions of the auxiliary space, whose time evolution is determined by a Hamiltonian obeying the constraint rigorously. Thus it is expected to be maintained as long as justifiable approximations are used.

In the zeroth order, the spinon field is diagonalized by applying the Bogoliubov transformation [7]: $s_{\sigma}(\mathbf{k})=\cosh \left(\xi_{\mathbf{k}}\right) \zeta_{\sigma}(\mathbf{k})+\sinh \left(\xi_{\mathbf{k}}\right) \zeta_{-\sigma}^{\dagger}(-\mathbf{k})$. The "bare" spinons, created by $\zeta_{\sigma}^{\dagger}(\mathbf{k})$, have energies $\epsilon^{\zeta}(\mathbf{k})$ with a V-shape zero minimum at $\mathbf{k}=\mathbf{k}_{0}$. Bose condensation results in $\mathrm{AF}$ order at wave vector $\mathbf{Q}=2 \mathbf{k}_{0}$.

Along the one dimensional charged stripes the spin-charge separation approximation is expected to be valid. Thus it is justified there to decouple two-particle spinon-holon (spinon-excession) Green's functions into singleauxiliary-particle Green's functions, and to interpret the auxiliary particles as physical quasiparticles. We refer to holons (excessions) along the charged stripes as "stripons", created by $p_{\mu}^{\dagger}(\mathbf{k})$, having bare energies by $\epsilon_{\mu}^{p}(\mathbf{k})$, and carrying charge $-\mathrm{e}$.

Since the dynamical stripe-like inhomogeneities are highly disordered, we find it appropriate to assume zeroth order localized stripon states. Their $\mathbf{k}$ wavenumbers present $\mathbf{k}$-symmetrized combinations of degenerate localized states to be treated in a perturbation expansion. Their values are within a Brillouin zone (BZ) based on periodic supercells which are large enough to approximately contain (each) the entire spectrum $\epsilon_{\mu}^{p}$ of bare stripon energies. These supercells introduce an approximate long-range order in spite of the local inhomogeneities.

We do not assume spin-charge separation away from the charged stripes, and construct approximate fermion creation operators of coupled holonspinon and excession-spinon basis states as follows:

$$
\begin{aligned}
f_{\lambda \sigma}^{\dagger}\left(\mathbf{k}^{\prime}, \mathbf{k}\right) & =e_{\lambda}^{\dagger}\left(\mathbf{k}^{\prime}\right) s_{\lambda,-\sigma}\left(\mathbf{k}^{\prime}-\mathbf{k}\right) / \sqrt{n_{\lambda}^{e}\left(\mathbf{k}^{\prime}\right)+n_{\lambda,-\sigma}^{s}\left(\mathbf{k}^{\prime}-\mathbf{k}\right)} \\
g_{\lambda \sigma}^{\dagger}\left(\mathbf{k}^{\prime}, \mathbf{k}\right) & =\sigma h_{\lambda}\left(\mathbf{k}^{\prime}\right) s_{\lambda \sigma}^{\dagger}\left(\mathbf{k}-\mathbf{k}^{\prime}\right) / \sqrt{n_{\lambda}^{h}\left(\mathbf{k}^{\prime}\right)+n_{\lambda \sigma}^{s}\left(\mathbf{k}-\mathbf{k}^{\prime}\right)}
\end{aligned}
$$


where the index $\lambda$ accounts for the structure of the unit cell, and: $n_{\lambda}^{e}(\mathbf{k}) \equiv$ $\left\langle e_{\lambda}^{\dagger}(\mathbf{k}) e_{\lambda}(\mathbf{k})\right\rangle, n_{\lambda}^{h}(\mathbf{k}) \equiv\left\langle h_{\lambda}^{\dagger}(\mathbf{k}) h_{\lambda}(\mathbf{k})\right\rangle, n_{\lambda \sigma}^{s}(\mathbf{k}) \equiv\left\langle s_{\lambda \sigma}^{\dagger}(\mathbf{k}) s_{\lambda \sigma}(\mathbf{k})\right\rangle$.

The states created by $f_{\lambda \sigma}^{\dagger}\left(\mathbf{k}^{\prime}, \mathbf{k}\right)$ and $g_{\lambda \sigma}^{\dagger}\left(\mathbf{k}^{\prime}, \mathbf{k}\right)$ are orthogonalized to the stripon states, and depleted to avoid over-completeness. Together with the small- $U$ states [created by $\left.c_{\nu \sigma}^{\dagger}(\mathbf{k})\right]$ they form, within the auxiliary space, a basis to "quasi-electron" (QE) states whose creation operators are denoted by $q_{\iota \sigma}^{\dagger}(\mathbf{k})$, and mean-field bare energies by $\epsilon_{l}^{q}(\mathbf{k})$. These energies form quasicontinuous ranges of bands within the $\mathrm{BZ}$ around $E_{\mathrm{F}}$.

The hopping and hybridization terms of the Hamiltonian introduce strong coupling between the QE, stripon and spinon fields. This can be expressed through a coupling Hamiltonian term whose parameters can be in principle derived self-consistently from the original Hamiltonian. For the case of p-type cuprates this coupling Hamiltonian can be expressed as:

$$
\begin{aligned}
\mathcal{H}^{\prime}= & \frac{1}{\sqrt{N}} \sum_{\iota \lambda \mu \sigma} \sum_{\mathbf{k}, \mathbf{k}^{\prime}}\left\{\sigma \epsilon _ { \iota \lambda \mu } ^ { q p } ( \mathbf { k } , \mathbf { k } ^ { \prime } ) q _ { \iota \sigma } ^ { \dagger } ( \mathbf { k } ) p _ { \mu } ( \mathbf { k } ^ { \prime } ) \left[\cosh \left(\xi_{\lambda, \mathbf{k}-\mathbf{k}^{\prime}}\right) \zeta_{\lambda \sigma}\left(\mathbf{k}-\mathbf{k}^{\prime}\right)\right.\right. \\
& \left.\left.+\sinh \left(\xi_{\lambda, \mathbf{k}-\mathbf{k}^{\prime}}\right) \zeta_{\lambda,-\sigma}^{\dagger}\left(\mathbf{k}^{\prime}-\mathbf{k}\right)\right]+h . c .\right\}
\end{aligned}
$$

where the $\mathbf{k}$ values correspond to the stripons $\mathrm{BZ}$, within which the other fields have been embedded. $\mathcal{H}^{\prime}$ introduces a vertex between the QE, stripon and spinon propagators [8].

A localized stripon modifies the lattice around it [9]. Consequently, physical process induced by $\mathcal{H}^{\prime}$, in which a stripon is transformed into a QE, or vice versa, and a spinon is emitted and/or absorbed, involves also the emission and/or absorption of phonons. This can be described by multiplying a spinon propagator, linked to the $\mathcal{H}^{\prime}$ vertex with a power series of phonon propagators [8]. Such a phonon- "dressed" spinon is referred to as a "svivon", carrying spin and lattice distortion, and the $\mathcal{H}^{\prime}$ vertex is re-interpreted as coupling between a QE, stripon, and svivon propagators.

\section{Auxiliary Spectral Functions}

The electrons spectral function are expressed in terms of auxiliary spectral functions $A_{\iota}^{q}(\mathbf{k}, \omega), A_{\mu}^{p}(\mathbf{k}, \omega)$, and $A_{\lambda}^{\zeta}(\mathbf{k}, \omega)$ of the QE's, stripons, and svivons, respectively $\left[A(\omega) \equiv \Im \mathcal{G}\left(\omega-i 0^{+}\right) / \pi\right.$, where $\mathcal{G}$ are the Green's functions]. Since the resulting stripon bandwidth is much smaller than the QE and svivon bandwidths, a phase-space argument as in the Migdal theorem results in negligible vertex corrections to the $\mathcal{H}^{\prime}$ vertex. Consequently $[6,8]$ the following expressions are obtained for the QE, stripon, and svivon scattering rates $\Gamma^{q}(\mathbf{k}, \omega), \Gamma^{p}(\mathbf{k}, \omega)$, and $\Gamma^{\zeta}(\mathbf{k}, \omega)\left[\Gamma(\mathbf{k}, \omega) \equiv 2 \Im \Sigma\left(\mathbf{k}, \omega-i 0^{+}\right)\right]$:

$$
\Gamma_{\iota \iota^{\prime}}^{q}(\mathbf{k}, \omega) \cong \frac{2 \pi}{N} \sum_{\lambda \mu \mathbf{k}^{\prime}} \int d \omega^{\prime} \epsilon_{\iota \lambda \mu}^{q p}\left(\mathbf{k}^{\prime}, \mathbf{k}\right) \epsilon_{\iota^{\prime} \lambda \mu}^{q p}\left(\mathbf{k}^{\prime}, \mathbf{k}\right)^{*} A_{\mu}^{p}\left(\mathbf{k}^{\prime}, \omega^{\prime}\right)
$$




$$
\begin{aligned}
& \times\left[-\cosh ^{2}\left(\xi_{\lambda, \mathbf{k}-\mathbf{k}^{\prime}}\right) A_{\lambda}^{\zeta}\left(\mathbf{k}-\mathbf{k}^{\prime}, \omega-\omega^{\prime}\right)\right. \\
& \left.+\sinh ^{2}\left(\xi_{\lambda, \mathbf{k}-\mathbf{k}^{\prime}}\right) A_{\lambda}^{\zeta}\left(\mathbf{k}-\mathbf{k}^{\prime}, \omega^{\prime}-\omega\right)\right]\left[f_{T}\left(\omega^{\prime}\right)+b_{T}\left(\omega^{\prime}-\omega\right)\right],(4) \\
\Gamma_{\mu \mu^{\prime}}^{p}(\mathbf{k}, \omega) & \cong \frac{2 \pi}{N} \sum_{\iota \mathbf{k}^{\prime} \sigma} \int d \omega^{\prime} \epsilon_{\iota \lambda \mu}^{q p}\left(\mathbf{k}^{\prime}, \mathbf{k}\right)^{*} \epsilon_{\iota \lambda \mu^{\prime}}^{q p}\left(\mathbf{k}^{\prime}, \mathbf{k}\right) A_{\iota}^{q}\left(\mathbf{k}^{\prime}, \omega^{\prime}\right) \\
& \times\left[\cosh ^{2}\left(\xi_{\lambda, \mathbf{k}^{\prime}-\mathbf{k}}\right) A_{\lambda}^{\zeta}\left(\mathbf{k}^{\prime}-\mathbf{k}, \omega^{\prime}-\omega\right)\right. \\
& \left.-\sinh ^{2}\left(\xi_{\lambda, \mathbf{k}^{\prime}-\mathbf{k}}\right) A_{\lambda}^{\zeta}\left(\mathbf{k}^{\prime}-\mathbf{k}, \omega-\omega^{\prime}\right)\right]\left[f_{T}\left(\omega^{\prime}\right)+b_{T}\left(\omega^{\prime}-\omega\right)\right],(5) \\
\Gamma_{\lambda \lambda^{\prime}}^{\zeta}(\mathbf{k}, \omega) & \cong \frac{2 \pi}{N} \sum_{\iota \mathbf{k}^{\prime} \mu} \int d \omega^{\prime} \epsilon_{\iota \lambda \mu}^{q p}\left(\mathbf{k}^{\prime}, \mathbf{k}^{\prime}-\mathbf{k}\right)^{*} \epsilon_{\iota \lambda^{\prime} \mu}^{q p}\left(\mathbf{k}^{\prime}, \mathbf{k}^{\prime}-\mathbf{k}\right) \\
& \times\left[\cosh \left(\xi_{\lambda \mathbf{k}}\right) \cosh \left(\xi_{\lambda^{\prime} \mathbf{k}}\right) A_{\iota}^{q}\left(\mathbf{k}^{\prime}, \omega^{\prime}\right) A_{\mu}^{p}\left(\mathbf{k}^{\prime}-\mathbf{k}, \omega^{\prime}-\omega\right)\right. \\
& \left.+\sinh \left(\xi_{\lambda \mathbf{k}}\right) \sinh \left(\xi_{\lambda^{\prime} \mathbf{k}}\right) A_{\iota}^{q}\left(\mathbf{k}^{\prime},-\omega^{\prime}\right) A_{\mu}^{p}\left(\mathbf{k}^{\prime}-\mathbf{k}, \omega-\omega^{\prime}\right)\right] \\
& \times\left[f_{T}\left(\omega^{\prime}-\omega\right)-f_{T}\left(\omega^{\prime}\right)\right],
\end{aligned}
$$

where $f_{T}(\omega)$ and $b_{T}(\omega)$ are the Fermi and Bose distribution functions at temperature $T$.

A solution with low-energy singularities is obtained [6] for the intermediary energy range $(\gtrsim 0.02 \mathrm{eV})$, where energies above few tenths of an $\mathrm{eV}$ are cut off, introducing spurious logarithmic divergences at $\pm \omega_{c}$. Expressions are derived, where the dependencies on $\mathbf{k}$ and band indices are omitted for simplicity (all the coefficients are positive, and analyticity is restored within the low-energy range $\lesssim 0.02 \mathrm{eV}$ ):

$$
\begin{gathered}
A^{q}(\omega) \cong\left\{\begin{array}{l}
a_{+}^{q} \omega+b_{+}^{q}, \quad \text { for } \omega>0, \\
-a_{-}^{q} \omega+b_{-}^{q}, \text { for } \omega<0,
\end{array}\right. \\
A^{p}(\omega) \cong \delta(\omega), \\
A^{\zeta}(\omega) \cong \begin{cases}a_{+}^{\zeta} \omega+b_{+}^{\zeta}, & \text { for } \omega>0, \\
a_{-}^{\zeta} \omega-b_{-}^{\zeta}, & \text { for } \omega<0,\end{cases} \\
\frac{\Gamma^{q}(\omega)}{2 \pi} \cong\left\{\begin{array}{l}
c_{+}^{q} \omega+d_{+}^{q}, \quad \text { for } \omega>0, \\
-c_{-}^{q} \omega+d_{-}^{q}, \text { for } \omega<0,
\end{array}\right. \\
\frac{\Gamma^{p}(\omega)}{2 \pi} \cong\left\{\begin{array}{l}
c_{+}^{p} \omega^{3}+d_{+}^{p} \omega^{2}+e_{+}^{p} \omega, \text { for } \omega>0, \\
-c_{-}^{p} \omega^{3}+d_{-}^{p} \omega^{2}-e_{-}^{p} \omega, \text { for } \omega<0,
\end{array}\right. \\
\frac{\Gamma^{\zeta}(\omega)}{2 \pi} \cong\left\{\begin{array}{l}
c_{+}^{\zeta} \omega+d_{+}^{\zeta}, \text { for } \omega>0, \\
c_{-}^{\zeta} \omega-d_{-}^{\zeta}, \text { for } \omega<0,
\end{array}\right. \\
-\Re \Sigma^{q}(\omega) \cong \omega_{c}\left(c_{+}^{q}-c_{-}^{q}\right)+\left(d_{+}^{q} \ln \left|\frac{\omega-\omega_{c}}{\omega}\right|-d_{-}^{q} \ln \left|\frac{\omega+\omega_{c}}{\omega}\right|\right) \\
+\omega\left(c_{+}^{q} \ln \left|\frac{\omega-\omega_{c}}{\omega}\right|+c_{-}^{q} \ln \left|\frac{\omega+\omega_{c}}{\omega}\right|\right),
\end{gathered}
$$




$$
\begin{aligned}
&-\Re \Sigma^{p}(\omega) \cong\left[\frac{\omega_{c}^{3}}{3}\left(c_{+}^{p}-c_{-}^{p}\right)+\frac{\omega_{c}^{2}}{2}\left(d_{+}^{p}-d_{-}^{p}\right)+\omega_{c}\left(e_{+}^{p}-e_{-}^{p}\right)\right] \\
&+\omega\left[\frac{\omega_{c}^{2}}{2}\left(c_{+}^{p}+c_{-}^{p}\right)+\omega_{c}\left(d_{+}^{p}+d_{-}^{p}\right)+e_{+}^{p} \ln \left|\frac{\omega-\omega_{c}}{\omega}\right|\right. \\
&\left.+e_{-}^{p} \ln \left|\frac{\omega+\omega_{c}}{\omega}\right|\right]+\omega^{2}\left[\omega_{c}\left(c_{+}^{p}-c_{-}^{p}\right)+d_{+}^{p} \ln \left|\frac{\omega-\omega_{c}}{\omega}\right|\right. \\
&\left.-d_{-}^{p} \ln \left|\frac{\omega+\omega_{c}}{\omega}\right|\right]+\omega^{3}\left[c_{+}^{p} \ln \left|\frac{\omega-\omega_{c}}{\omega}\right|+c_{-}^{p} \ln \left|\frac{\omega+\omega_{c}}{\omega}\right|\right], \\
&-\Re \Sigma^{\zeta}(\omega) \cong \omega_{c}\left(c_{+}^{\zeta}+c_{-}^{\zeta}\right)+\left(d_{+}^{\zeta} \ln \left|\frac{\omega-\omega_{c}}{\omega}\right|+d_{-}^{\zeta} \ln \left|\frac{\omega+\omega_{c}}{\omega}\right|\right) \\
&+\omega\left(c_{+}^{\zeta} \ln \left|\frac{\omega-\omega_{c}}{\omega}\right|-c_{-}^{\zeta} \ln \left|\frac{\omega+\omega_{c}}{\omega}\right|\right) .
\end{aligned}
$$

The auxiliary-particle energies are renormalized: $\bar{\epsilon}=\epsilon+\Re \Sigma(\bar{\epsilon})$. This renormalization is particularly strong on the stripon energies where the bandwidth drops down to the low energy range.

For the discussed case of p-type cuprates the following inequalities between the coefficients in Eqs. (7-12) are generally expected [6]:

$$
\begin{array}{llll}
a_{+}^{q}>a_{-}^{q}, & b_{+}^{q}>b_{-}^{q}, & c_{+}^{q}>c_{-}^{q}, & d_{+}^{q}>d_{-}^{q}, \\
a_{+}^{\zeta}>a_{-}^{\zeta}, & b_{+}^{\zeta}>b_{-}^{\zeta}, & c_{+}^{\zeta}>c_{-}^{\zeta}, & d_{+}^{\zeta}>d_{-}^{\zeta} .
\end{array}
$$

For "real" n-type cuprates (namely, ones where the stripons are based on excession and not holon states) the direction of the inequalities is reversed for the QE coefficients (16).

\section{Consequences for Electron Spectroscopies}

In order to apply the auxiliary spectral functions in Eqs. (7-9) for physical properties, a projection into the physical space is required. The low-energy signature of the stripon spectral functions is not detected by spectroscopies, like ARPES, which measure the effect of transfer of electrons into, or out of, the crystal. It is smeared over few tenths of an $\mathrm{eV}$ through convolution with svivon spectral functions, and contributes an "incoherent" background. On the other hand, transport properties measure the electrons within the crystal, and can detect the stripon energy scale. The physical contribution of the quasi-continuum of $\mathrm{QE}$ bands to the electron spectral functions is strong only for few QE bands for which the expansion coefficients in terms of their basis states [created by $(1,2) f_{\lambda \sigma}^{\dagger}\left(\mathbf{k}^{\prime}, \mathbf{k}\right), g_{\lambda \sigma}^{\dagger}\left(\mathbf{k}^{\prime}, \mathbf{k}\right)$, and $c_{\nu \sigma}^{\dagger}(\mathbf{k})$ ] closely correspond to those of real electron states, and they contribute "coherent" $\bar{\epsilon}^{q}(\mathbf{k})$ bands. The other QE states also contribute to the incoherent background. The dependence of the QE basis states $(1,2)$ on $n_{\lambda}^{e}, n_{\lambda}^{h}$, and $n_{\lambda \sigma}^{s}$ is reflected in the observed dependence [10] of strongly correlated electrons spectrum on these occupation factors. 
The characteristics of the auxiliary spectral functions are maintained in their projection to the physical space (detected, e.g., by ARPES). The fact that the QE field is coupled to the stripon and svivon fields is reflected, e.g., in "shadow bands". The results for $\Gamma^{q}(10)$ are reflected in the observed nonFermi-liquid bandwidths, having a $\propto \omega$ and a constant term. The results for $\Re \Sigma^{q}(13)$ are reflected in the renormalization of band slopes, compared to LDA predictions. The consequence of the logarithmic singularity in $\Re \Sigma^{q}$ at $\omega=0$ [due to the $\left(d_{+}^{q}-d_{-}^{q}\right) \ln |\omega|$ term in Eq. (13)], for p-type cuprates (16), is that as an $\bar{\epsilon}^{q}(\mathbf{k})$ band approaches $E_{\mathrm{F}}$ from below it is becoming flatter (though it does not narrow accordingly) while from above it becomes steeper, and may pass through an infinite slope resulting in an S-shape triply valued band (causing smearing of the spectral weight, and blurring the band). The effect of the singularity is truncated and smoothened on an energy scale $\lesssim 0.02 \mathrm{eV}$. For "real" n-type cuprates these behaviors below and above $E_{\mathrm{F}}$ are expected to switch. ARPES data reflects the behavior below $E_{\mathrm{F}}$, and results of such band-flattening have been reported in p-type cuprates (and attributed to electron coupling with phonons [11] or with the neutron scattering resonance mode [12]). The behavior above $E_{\mathrm{F}}$ would be detected in ARIPES measurements. Further measurements of the bands of n-type cuprates very close to $E_{\mathrm{F}}$ are necessary to determine their behavior there.

The renormalization of svivon energies (from $\epsilon^{\zeta}$ to $\bar{\epsilon}^{\zeta}$ ) due to $\Re \Sigma^{\zeta}(\omega)$ (15), and specifically its logarithmic singularity at $\omega=0$ due to the $\left(d_{+}^{\zeta}+d_{-}^{\zeta}\right) \ln |\omega|$ term (which is smoothened at low energies) results in a negative shift close to the minimum at $\mathbf{k}_{0}$; and as $\mathbf{k}_{0}$ is approached, the slope of $\bar{\epsilon}^{\zeta}(\mathbf{k})$ increases first [compared to the almost constant slope of $\epsilon^{\zeta}(\mathbf{k})$ ] and it may become infinite, resulting in a range of an S-shape triply valued and blurred band; but as $\mathbf{k}_{0}$ is further approached, the slope of $\bar{\epsilon}^{\zeta}(\mathbf{k})$ decreases and becomes smaller than the slope of $\epsilon^{\zeta}(\mathbf{k})$ at its minimum at $\mathbf{k}_{0}$. From analyticity arguments (due to the absence of long-range AF order) it is expected that $\bar{\epsilon}^{\zeta}(\mathbf{k})$ has a smooth minimum, rather than the V-shape minimum of $\epsilon^{\zeta}(\mathbf{k})$. Thus $-\bar{\epsilon}^{\zeta}\left(\mathbf{k}_{0}\right)$ presents the crossover energy from the intermediary to the low energy range. Since spin-flip excitations are, largely, double-svivon absorption or emission processes, $-2 \bar{\epsilon}^{\zeta}\left(\mathbf{k}_{0}\right)$ is expected to be the energy of such an excitation at $\mathbf{k}=\mathbf{Q}$. This result is consistent with the neutron-scattering resonance found at this wave number [13]. It is observed that this resonance is at a local energy maximum, for $\mathbf{k}$ around $\mathbf{Q}$, consistently with our result that $\bar{\epsilon}^{\zeta}\left(\mathbf{k}_{0}\right)$ is at a minimum below zero.

An anomalous optical conductivity has been observed for the high- $T_{c}$ cuprates [14], consisting of Drude and mid-IR terms. This is consistent with our results, where the Drude term is due to transitions between QE states, and between stripon states, while the mid-IR term largely results 
from transitions between stripon states and QE+svivon states. The mid-IR term becomes negligibly small for $\omega \rightarrow 0$, where the conductivity could be decoupled into QE and stripon terms.

\section{Transport Properties}

Normal-state transport expressions [not including the effect of the pseudogap (PG)] are obtained [6] using linear response theory, where the zero-energy singularities in the auxiliary spectral functions and (7-9) are smoothened in the low-energy scale [and $\left.A^{\zeta}(\omega=0)=0\right]$. Also it is taken into account that the electric current can be expressed as a sum $\mathbf{j}=\mathbf{j}_{0}^{q}+\mathbf{j}_{0}^{p}$ of contributions of "bare" $\mathrm{QE}$ and and stripon states, where $\mathbf{j}_{0}^{p} \cong 0$ since the bare stripon states are localized.

Results for the electrical resistivity $\rho$, the Hall constant $R_{\mathrm{H}}$, the Hall number $n_{\mathrm{H}}=1 / \mathrm{e} R_{\mathrm{H}}$, the Hall angle $\theta_{\mathrm{H}}$ (through $\left.\cot \theta_{\mathrm{H}}=\rho / R_{\mathrm{H}}\right)$, and the thermoelectric power (TEP) $S$, are presented in Fig. 1. Their anomalous temperature dependencies result both from the low energy scale stripon band (8), modeled here by a "rectangular" shape $A^{p}$ of width $\omega^{p}$ and fractional occupation $n^{p}$, and from those of the scattering rates $\Gamma^{q}(T, \omega=0)$ and $\Gamma^{p}(T, \omega=0)$, derived using Eqs. $(4,5)$ to which temperature independent terms are added to account for impurity scattering.

The transport results in Fig. 1 correspond to five stoichiometries, ranging from underdoped $\left(n^{p}=0.8\right)$ to overdoped $\left(n^{p}=0.4\right)$ p-type cuprates. The parameter $N_{e}^{q}$, corresponding to the QE contribution to the electrons density of states at $E_{\mathrm{F}}$, is assumed to increase with doping, reflecting transfer of $\mathrm{QE}$ spectral weight towards $E_{\mathrm{F}}$. Consequently (5) $\omega^{p}$ is assumed to decrease with doping. The parameters $n_{\mathrm{H}}^{q}$ and $n_{\mathrm{H}}^{p}$ represent effective $\mathrm{QE}$ and stripon contributions to the density of charge carriers (reflected in the Hall number). Since they both contribute through the current $\mathbf{j}_{0}^{q}$ of the bare QE states, they are expected to have same sign (corresponding to these states). The values of these parameters are assumed to increase with doping, where $n_{\mathrm{H}}^{p}$ increases considerably faster than $n_{\mathrm{H}}^{q}$, and a little faster than $1-n^{p}$ (since $n_{\mathrm{H}}^{p}$ reflects an overall stripon-related carriers density, while $n^{p}$ is the fractional occupation of stripon states within the charged stripes whose number increases with doping). Doping-independent values are taken for the QE TEP parameter $S_{1}^{q}$ [which is negative for p-type cuprates by the inequality (16)], and for the stripon and QE scattering rate parameters $\gamma_{0}^{p}$, $\gamma_{2}^{p}, \gamma_{0}^{q}$, and $\gamma_{1}^{q}$.

The TEP results depend strongly on $n^{p}$, and reproduce very well the doping-dependent experimental behavior $[15,16,17]$. The position of the maximum in $S$ depends on the choice of $\omega^{p}$, and it may occur below or above $T_{c}$ (a PG may shift it to a higher temperature than predicted here). Also the 
other transport coefficient in Fig. 1 reproduce very well the experimental behavior $[18,19]$. Cases where the linear $T$ dependence of the resistivity persists at very low temperatures are expected as a PG effect.

The TEP in "real" n-type cuprates is expected to behave similarly to p-type cuprates, but with an opposite sign and slope. Results for NCCO [20] reveal that for doping levels where it is superconducting (SC), the TEP slope actually corresponds to those of p-type cuprates [Fig. 1(a)], but with an opposite effect of doping. Thus $S$ for lower doping levels is typical of the p-type overdoped regime. This points to the possibility that NCCO is not a "real" n-type cuprate, and its stripons are also based on holon states, where the extra doped negative charge occupies another QE band. The Hall constant in NCCO [20] is also consistent with this scenario and our results. Another possibility is that the TEP slope in NCCO is determined by electrons which are not strongly coupled to the $\mathrm{CuO}_{2}$ planes.

\section{Mechanism - BCS-BEC Crossover}

The electronic structure of the cuprates discussed here provides SC pairing due to transitions between pair states of stripons and QE's through the exchange of svivons [8]. Since such transitions enable long-range hopping of local stripon pairs, without an associated hopping of svivons (necessary for single-stripon hopping), this type of pairing results in a large gain in the "kinetic" energy of the stripons. Thus this can effectively be expressed as a strong attractive stripon-stripon interaction. Thus, pair-breaking is then expected to result in both QE and stripon+svivon excitations. This is confirmed by ARPES results in the SC state [21], showing a sharp peak at $\sim 0.04 \mathrm{eV}$ over a wide range of the BZ, and a "hump" starting at $\sim 0.1 \mathrm{eV}$ and merging with a normal-state band at higher energies, corresponding to these excitations. The hump is consistent with the QE pair-breaking excitation, and the peak with the stripon+svivon pair-breaking excitation at the svivon minimum $\bar{\epsilon}^{\zeta}\left(\mathbf{k}_{0}\right)$. In the $\mathrm{SC}$ state this minimum is within the gap, and thus is considerably narrower than in the normal state [reflected also in the width of the neutron-scattering resonance energy $\left.-2 \bar{\epsilon}^{\zeta}\left(\mathbf{k}_{0}\right)\right]$.

When the pairing energy is comparable to the relevant bandwidth, a crossover is expected [22] to occur between BCS and preformed-pairs BoseEinstein condensation (BEC) behaviors. This condition is fulfilled here due to the small stripon bandwidth, and the underdoped cuprates are characterized by BEC behavior, where singlet pairs are formed at $T_{\text {pair }}$, and $\mathrm{SC}$ occurs below $T_{\text {coh }}\left(<T_{\text {pair }}\right)$ where phase coherence sets in. The normal-state PG is a pair-breaking gap at $T_{\text {coh }}<T<T_{\text {pair }}$, having similarities to the SC gap, and accounting for most of the pairing energy, as observed [23]. The coherence temperature can be expressed [24] as: $T_{\text {coh }} \propto n_{s} / m_{s}^{*}$, where 
$m_{s}^{*}$ and $n_{s}$ are the pairs effective mass and density, in agreement with the "Uemura plots". Since we expect [Fig. 1(a)] the stripon band to be half full $\left(n^{p}=\frac{1}{2}\right)$ for slightly overdoped cuprates, the "boomerang-type" behavior [25] of these plots is understood as a crossover between a stripon band-top

$T_{c}=T_{\text {coh }}$ behavior in the underdoped cuprates, and a stripon band-bottom $T_{c}=T_{\text {pair }}$ behavior in overdoped cuprates.

\section{Conclusions}

It was shown that a treatment of the cuprates on the basis of both large$U$ and small- $U$ orbitals and dynamical stripe-like inhomogeneities resolves puzzling normal-state spectroscopic and transport properties and provides a mechanism for high- $T_{c}$ and the peudogap.

\section{References}

1. J. Zaanen, and O. Gunnarsson, Phys. Rev. B 40, 7391 (1989).

2. V. J. Emery, and S. A. Kivelson, Physica C 209, 597 (1993).

3. Papers in Int. J. Mod. Phys. 14, 3289-3790 (2000).

4. O. K. Andersen et al., J. Phys. Chem. Solids 56, 1573 (1995).

5. S. E. Barnes, Adv. Phys. 30, 801 (1980).

6. J. Ashkenazi, J. Phys. Chem. Solids (2002); cond-mat/0108383.

7. J. Ashkenazi, J. Supercond. 7, 719 (1994).

8. J. Ashkenazi, High-Temperature Superconductivity, edited by S. E. Barnes, J. Ashkenazi, J. L. Cohn, and F. Zuo (AIP Conference Proceedings 483, 1999), p. 12; condmat/9905172.

9. A. Bianconi, et al., Phys. Rev. B 54, 12018 (1996).

10. H. Eskes, et al., Phys. Rev. Lett. 67, 1035 (1991).

11. Z. X. Shen, et al., cond-mat/0108381.

12. P. D. Johnson, et al., Phys. Rev. Lett. 87, 177007 (2001).

13. H. F. Fong, et al., Phys. Rev. B 61, 14773 (2000).

14. D. B. Tanner, and T. Timusk, Physical Properties of High Temperature Superconductors III, edited by D. M. Ginsberg (World Scientific, 1992), p. 363.

15. B. Fisher, et al., J. Supercond. 1, 53 (1988); J. Genossar, et al., Physica C 157, 320 (1989).

16. S. Tanaka, et al., J. Phys. Soc. Japan 61, 1271 (1992).

17. K. Matsuura, et al., Phys. Rev. B 46, 11923 (1992); S. D. Obertelli, et al., ibid., p. 14928; C. K. Subramaniam, et al., Physica C 203, 298 (1992).

18. Y. Kubo and T. Manako, Physica C 197, 378 (1992).

19. H. Takagi, et al., Phys. Rev. Lett. 69, 2975 (1992); H. Y. Hwang, et al., ibid. 72, 2636 (1994).

20. J. Takeda, et al., Physica C 231, 293 (1994); X.-Q. Xu, et al., Phys. Rev. B 45, 7356 (1992); Wu Jiang, et al., Phys. Rev. Lett. 73, 1291 (1994).

21. M. R. Norman, and H. Ding, Phys. Rev. B 57, R11089 (1998).

22. M. Randeria, cond-mat/9710223, Varenna Lectures (1997); J. R. Engelbrecht, et al., Phys. Rev. B 55, 15153 (1997); R. D. Duncan, and C. A. R. Sá de Melo, ibid 62, 9675 (2000); Q. Chen, et al., ibid 63, 184159 (2001).

23. C. P. Moca, and B. Jankó, Phys. Rev. B 65, 052503 (2002).

24. V. J. Emery, and S. A. Kivelson, Nature 374, 4347 (1995).

25. Ch. Niedermayer, et al., Phys. Rev. Lett. 71, 1764 (1993). 

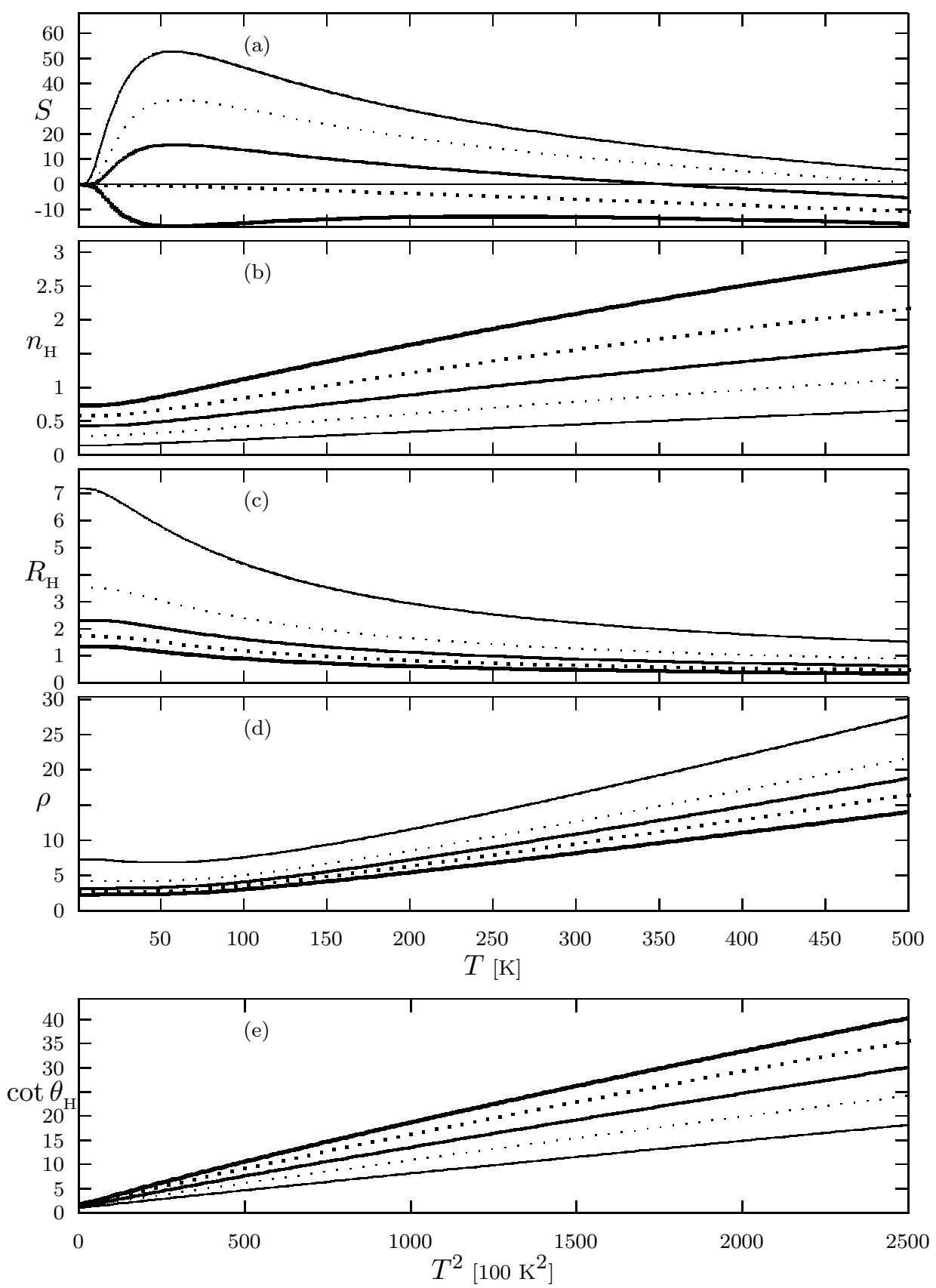

Figure 1. The transport coefficients, in arbitrary units [and $\mu \mathrm{V} / \mathrm{K}$ units for $S$ (a)], for: $n^{p}=0.8,0.7,0.6,0.5,0.4 ; 10000 N_{e}^{q}=20,23,26,29,32 ; \quad \omega^{p}[\mathrm{~K}]=200,190,180,170,160$; $n_{\mathrm{H}}^{p}=0.1,0.2,0.3,0.4,0.5 ; n_{\mathrm{H}}^{q}=6,7,8,9,10 ; S_{1}^{q}=-0.025 ; \gamma_{0}^{p}=500 ; \gamma_{2}^{p}=0.03 ; \gamma_{0}^{q}=5 ; \gamma_{1}^{q}=0.2$. The last values correspond to the thickest lines. 